\title{
INVESTIGATION OF THE ARC-ANODE ATTACHMENT AREA BY UTILIZING A HIGH-SPEED CAMERA
}

\author{
OndaC P. ${ }^{a, b, *}$, Maslani A. ${ }^{a}$, Hrabovsky M. ${ }^{a}$ \\ ${ }^{a}$ Institute of Plasma Physics AS CR, Za Slovankou 1782/3, 18200 Prague 8, Czech Republic \\ ${ }^{b}$ Department of Surface and Plasma Science, Faculty of Mathematics and Physics, Charles University, \\ V Holesovickach 2, 18000 Prague 8, Czech Republic \\ * ondac@ipp.cas.cz
}

\begin{abstract}
The arc-anode attachment in the DC plasma arc influences power distribution in the plasma, a lifespan of anode and flow structure of plasma jet. A movement of the attachment and the surrounding plasma was directly observed by using a high-speed camera (max. $1080000 \mathrm{fps}$ ). The observations were compared with cathode-anode voltage measurements (sample rate $80 \mathrm{MHz}$ ). We have directly measured the average velocity of the attachments and hydrodynamic waves above them, as well as the characteristic dwell times and dwell frequencies of the attachments.
\end{abstract}

Keywords: plasma, arc, anode, attachment, camera, wave.

\section{Introduction}

The hybrid water-gas DC arc plasma torch [1] is used for plasma spraying [2] or bio-waste gasification [3. Transferred plasma torches are also used for plasma arc cutting [4] and welding [5]. An electric arc burns between an internal cathode and, consisting of a cooper disk located downstream of the exit nozzle, an external anode. The arc anode processes affect the properties of generated plasma jet (visible part of the plasma flow) and the lifespan of the anode.

In this work, the so-called restrike anode mode [6[10] is studied. For this mode, the anode attachment moves periodically downstream along the upper anode surface. The motion of this anode attachment is still not a well understood phenomenon. Especially, mechanisms that control the motion of the anode attachment and process of reigniting the anode attachment in the upstream part of the anode surface. It is assumed that the position of the anode attachment is determined by the balance of the gas dynamic drag force acting in the downstream direction and the Lorentz force acting in the upstream direction due to the double curvature of the anode attachment [11, 12].

The purpose of this work is to show new and detailed experimental observations of the an-ode attachment area in the restrike mode. For this purpose, we have used a high-speed camera. For the first time, the anode attach-ment area was observed by using a frame rate of $300000 \mathrm{fps}$ and $1080000 \mathrm{fps}$ with an exposure time of $0.25 \mu \mathrm{s}$ and $0.29 \mu \mathrm{s}$.

\section{Experiments}

We have performed our measurements on a plasma torch (Fig. 1) with the DC electric arc stabilized by water vortex and argon [1].

The rotating copper anode and $2 \%$ thoriated tungsten cathode are cooled down by a separate water

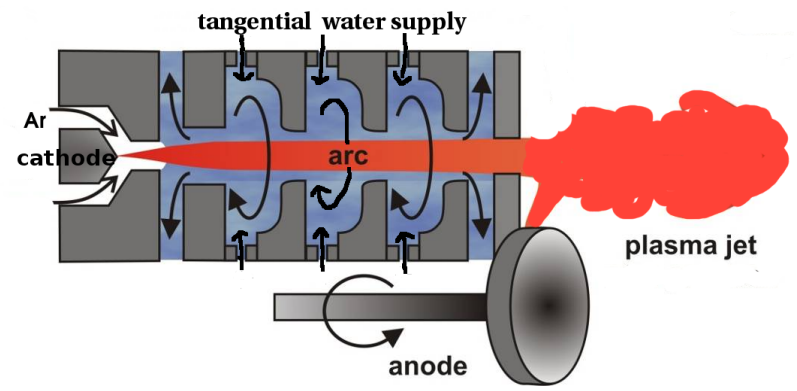

Figure 1. Sketch of the plasma torch with DC electric arc stabilized by the water vortex and argon

circuit. During rotation, the anode surface moves perpendicular to the main stream of the plasma jet with the velocity around $47 \mathrm{~m} / \mathrm{s}$. The thickness of the anode is $(16 \pm 1) \mathrm{mm}$ and the vertical distance between the bottom edge of the gap (with diameter about $6 \mathrm{~mm}$ ) in the exit nozzle and the upper anode surface was around $0.8 \mathrm{~mm}$. The horizontal distance between the exit nozzle and the anode was about $4.5 \mathrm{~mm}$. The arc current is kept constant and the cathode-anode voltage periodically changes in sawtooth form, mainly because of the periodical movement of the anode attachment. In all cases mentioned in this work, the plasma jet was at atmospheric pressure.

We have filmed the arc-anode attachment area by using the monochromatic high-speed camera Photron FASTCAM SA-X2. Because of strong radiation and the brightness of the plasma jet, an optical filter in front of the camera objective was needed. We have used the optical filter with $\mathrm{CWL}=809 \mathrm{~nm}$ and $\mathrm{FWHM}=12.5 \mathrm{~nm}$. The detailed image of the anode attachment area taken by this camera with the optical filter is shown in Fig. 2 (visible spectral lines originate mainly from argon atoms).

We have also measured the cathode-anode voltage 


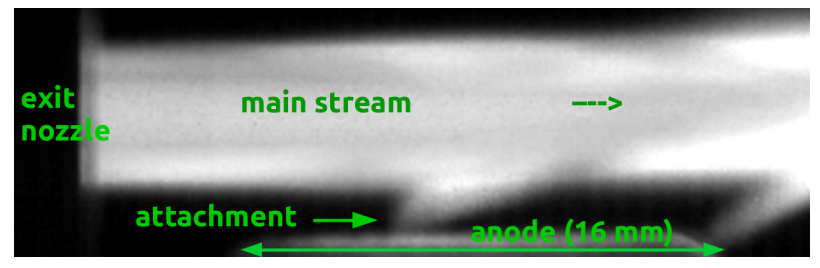

Figure 2. The arc-anode attachment area detail; We see 2 attachments and radiation has wavelength of $(810 \pm 12) n m$; Exposure time $0.29 \mu \mathrm{s}$

with a sampling frequency of $80 \mathrm{MHz}$, by using the high voltage probe Tektronix P6015 connected to a PC Oscilloscope (PicoScope). This probe decreased the voltage signal 1000 times (the maximum ignition voltage was around $5 \mathrm{kV}$ ). The videos were synchronized with the cathode-anode voltage measurements with the help of the synchronized signal from the camera to the PicoScope. The whole experimental setup is illustrated in Fig. 3 With the help of three isolation transformers, the background electrical noise was only $\pm 0.3 \mathrm{~V}$.

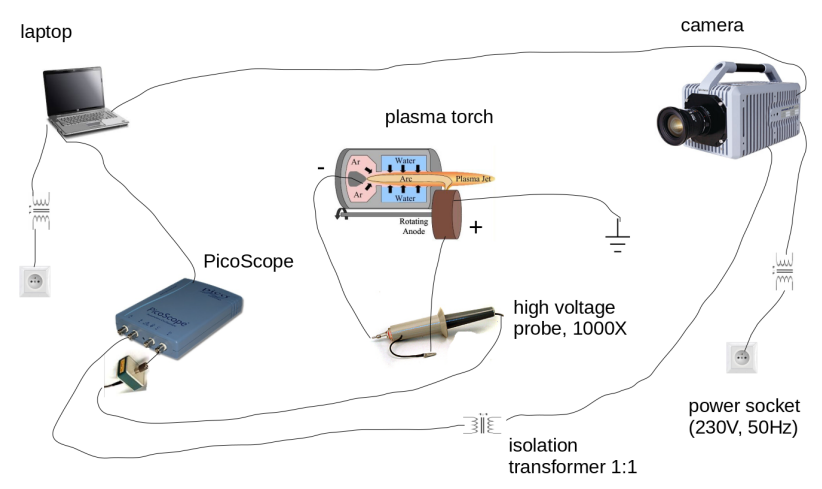

Figure 3. The experimental setup used

\section{Results}

We have investigated the arc-anode attachment area under 6 combinations of the plasma torch parameters: three values of the arc current (300 A, 400 A and $500 \mathrm{~A}$ ) and two values of the argon flow rate $(12.5 \mathrm{slm}$ and $22.5 \mathrm{slm})$.

Firstly, we have directly measured the velocities of the anode attachments defined by bright connections between the main stream of the plasma and the anode surface, see Fig. 2 (electric current flows through a part of these bright connections). We have observed more than one attachment in one frame and they interacted each other, sometimes.

We have also directly measured wavelengths and velocities of the hydrodynamic waves in the plasma jet (see Fig. 4). These waves are the result of KelvinHelmholtz instability and arise because of the velocity and density gradient between the low density, fast plasma jet and high density, steady ambient atmosphere.
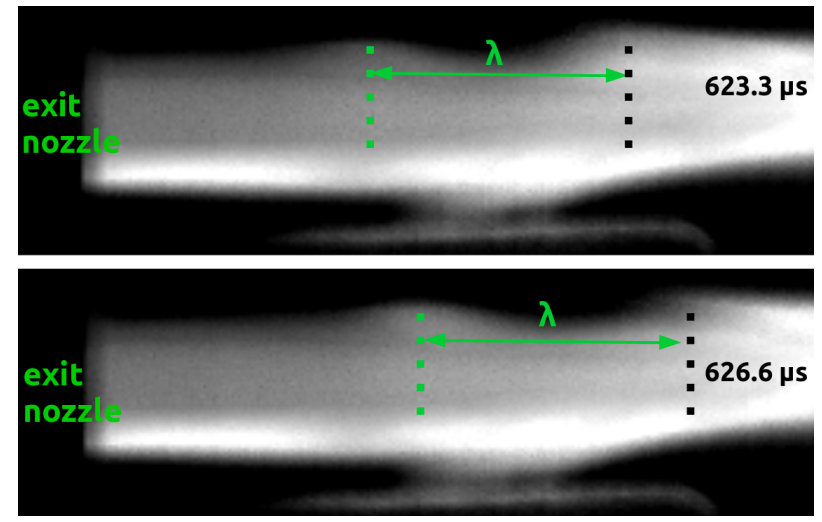

Figure 4. The hydrodynamic wave in the plasma jet in 2 consecutive frames with exposure time $0.29 \mu \mathrm{s}$; The downstream direction is to the right; Visible radiation has wavelength of $(810 \pm 12) \mathrm{nm}$

The velocities of the attachments and waves were calculated from the images by recording their positions frame by frame (frame rate $300000 \mathrm{fps}, 256 \times 80$ pixel resolution), with respect to time. Their positions were defined as the horizontal position of the upstream edge (closer to the exit nozzle) in the case of attachments, and as the horizontal position of one crest in the series of waves.

The position vs. time graphs (for attachments are shown in Fig. 5 b.) were approximated by the linear trend line and the average velocities were calculated as an average value of the slopes of these linear trend lines. We computed the uncertainties of the slopes with known uncertainty on position data (due to image resolution) and combined them (by the summation in quadrature) with the standard uncertainty of the mean. The total standard uncertainties of the velocities are listed in Table 1.

In the case of the attachments, we omitted the position vs. time data in anode edge area (more than $\approx 14 \mathrm{~mm}$ in position) because in this area the anode surface is curved.

The hydrodynamic waves start to become clearly visible just above the anode. This is probably due to the vapours of the anode material that amplify them. Especially they are strongly amplified when they propagate just above the attachment. Their wavelength (distance between two consecutive crests) above the anode was determined from several waves for each combination of the torch parameters. It was $(7 \pm 1) \mathrm{mm}$. This wave-length seems to be independent of the arc current and the argon flow rate and only slightly increases with the corresponding distance from the exit nozzle.

In Fig. 5 b, we can see the typical behaviour of the anode attachments. They periodically move and stop at certain positions on the anode surface. We also calculated characteristic dwell times and characteristic dwelling frequencies for the attachments. The characteristic dwell time is meant to be the average time during which the attachment dwells/stops at a 


\begin{tabular}{llllcl}
\hline \hline $\begin{array}{l}\text { Argon } \\
\text { flow rate } \\
\pm 0.5 \mathrm{slm}\end{array}$ & $\begin{array}{l}\text { Arc } \\
\text { current } \\
\pm 2 \mathrm{~A}\end{array}$ & $\begin{array}{l}\text { Average } \\
\text { attachment } \\
\text { velocity }\end{array}$ & $\begin{array}{l}\text { Average wave } \\
\text { velocity above } \\
\text { the anode }\end{array}$ & $\begin{array}{l}\text { Characteristic } \\
\text { dwell time for } \\
\text { attachment }\end{array}$ & $\begin{array}{l}\text { Characteristic } \\
\text { dwelling } \\
\text { frequency } \pm 2 ~ k H z\end{array}$ \\
\hline $12.5 \mathrm{slm}$ & $300 \mathrm{~A}$ & $85 \pm 3 \mathrm{~m} / \mathrm{s}$ & $370 \pm 50 \mathrm{~m} / \mathrm{s}$ & $14 \pm 3 \mu \mathrm{s}$ & $5 \mathrm{kHz}$ \\
$12.5 \mathrm{slm}$ & $400 \mathrm{~A}$ & $146 \pm 9 \mathrm{~m} / \mathrm{s}$ & $560 \pm 60 \mathrm{~m} / \mathrm{s}$ & $17 \pm 3 \mu \mathrm{s}$ & $10 \mathrm{kHz}$ \\
$12.5 \mathrm{slm}$ & $500 \mathrm{~A}$ & $190 \pm 11 \mathrm{~m} / \mathrm{s}$ & $680 \pm 90 \mathrm{~m} / \mathrm{s}$ & $22 \pm 5 \mu \mathrm{s}$ & $9 \mathrm{kHz}$ \\
$22.5 \mathrm{slm}$ & $300 \mathrm{~A}$ & $99 \pm 8 \mathrm{~m} / \mathrm{s}$ & $470 \pm 40 \mathrm{~m} / \mathrm{s}$ & $13 \pm 3 \mu \mathrm{s}$ & $15 \mathrm{kHz}$ \\
$22.5 \mathrm{slm}$ & $400 \mathrm{~A}$ & $215 \pm 6 \mathrm{~m} / \mathrm{s}$ & $650 \pm 50 \mathrm{~m} / \mathrm{s}$ & $14 \pm 3 \mu \mathrm{s}$ & $17 \mathrm{kHz}$ \\
$22.5 \mathrm{slm}$ & $500 \mathrm{~A}$ & $298 \pm 14 \mathrm{~m} / \mathrm{s}$ & $800 \pm 70 \mathrm{~m} / \mathrm{s}$ & $14 \pm 3 \mu \mathrm{s}$ & $15 \mathrm{kHz}$ \\
\hline \hline
\end{tabular}

Table 1. The average velocities, characteristic dwell times and dwelling frequencies (green cells) calculated for 6 combinations of the plasma torch parameters (first 2 columns)

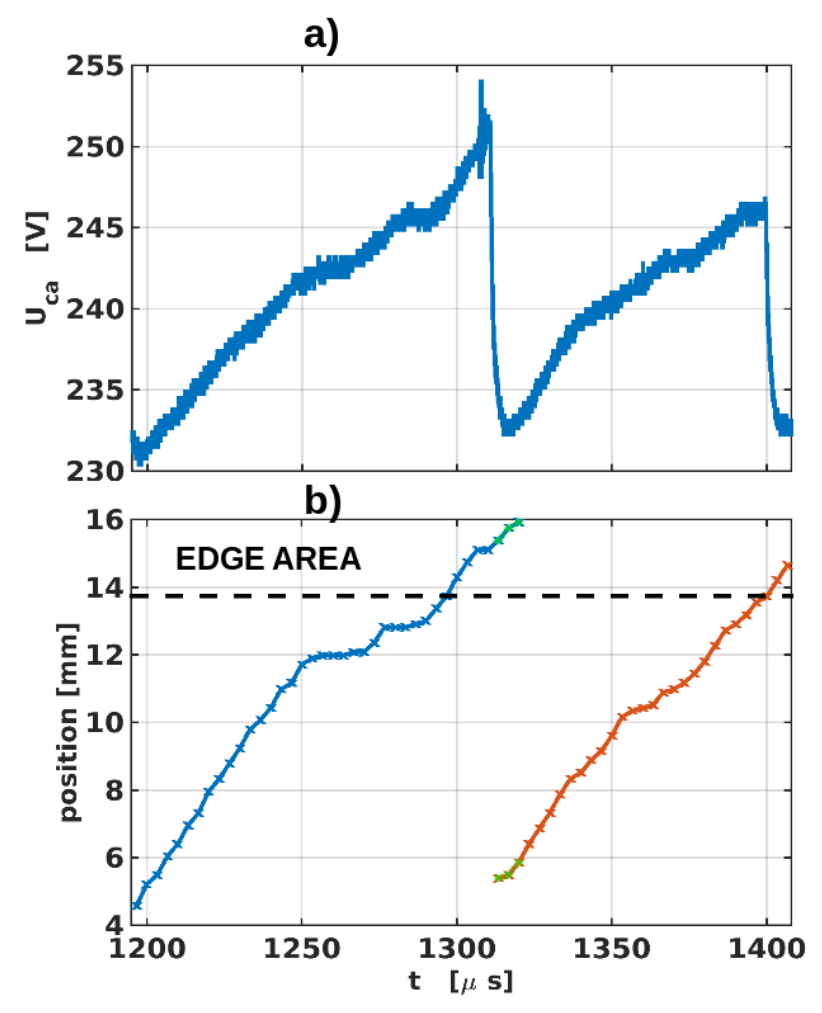

Figure 5. The synchronized cathode-anode voltage time dependence - raw data (a) and typical positiontime dependencies for 2 attachments (b)

certain position of the anode surface. The average frequency with which the attachments come to a stop is called the characteristic dwelling frequency, in this work. We have taken into consideration only dwellings of the attachments longer than approx. 4 us (it defines the stop of the attachment).

The calculated quantities for 6 mentioned combinations of the plasma torch parameters are shown in Table 1. Every value of the average attachment/wave velocity was obtained from 8 attachments/waves. Every value of the characteristic dwell time and dwelling frequency was obtained from the time interval $2 \mathrm{~ms}$ of the cathode-anode voltage time dependencies (after removing frequencies higher than $1 \mathrm{MHz}$ by using the fast Fourier transform). In these voltage vs. time graphs (see Fig. 5 a) the dwelling of the attachments is visible as a constant value of the voltage for a short time. The sampling frequency $80 \mathrm{MHz}$ is much greater than in the case of the position vs. time graphs $(300 \mathrm{kHz})$. Sometimes, there were also small drops in voltage, what can be explained by an upstream movement of the conductive path within the stopped attachment.

\section{Discussion and conclussions}

The arc-anode attachments very often come to a stop at certain positions of the anode surface. We think that these positions are anode surface inhomogeneities, where anode material vaporizes faster than in the immediate vicinity. During dwelling of attachments at these inhomogeneities, the anode material vaporizes faster than during movement of attachments. Thus, an anode with more homogenous surface should have the longer lifespan than the anode with less homogenous surface. The vapours of the anode material move downstream because of the plasma flow, and the anode attachments incline downstream.

We have not observed the second curvature of the anode attachment, close to the anode surface, proposed in the previous publications [11, 12. It can be very small and therefore not easily visible even in our detailed images. In the upstream direction, a different magnetic force, caused by electromagnetic induction, can act on the induced electric current. As the anode attachment moves downstream, the magnetic flux in the area between the electric arc and the anode increases with time, and the opposite electric current (decreasing the total electric current) should be induced within the electric arc.

The average anode attachment velocity and hydrodynamic wave velocity increases with the arc current as well as with the argon flow rate (Table 1 ). The greater arc current causes faster water evaporation rate. The faster evap-oration rate or faster argon flow rate causes greater pressure in the cavity of the plasma torch and consequently faster flowing of the plasma jet. We have confirmed our calculations of the attachment velocities also in the video with frame rate $1080000 \mathrm{fps}$ and exposure time $0.25 \mu \mathrm{s}$, however with a worse pixel resolution $(128 \times 8)$ and consequently 
greater uncertainties.

The velocities, wavelengths and corresponding frequencies of the waves, obtained first time directly, by using the high-speed camera, correspond with previously indirectly obtained velocities, wavelengths and frequencies of hydrodynamic waves, measured by using an array of high frequency photodiodes [13-16]. The waves above the attachment move around 7-8times slower than the plasma flow itself [17].

The characteristic dwell time for the anode attachments only slightly increased with the arc current and the argon flow rate. The characteristic dwelling frequency for the attachments increases with the arc current and with the argon flow rate for small plasma velocities (the faster the attachment moves, the more often it comes to a stop). However, too fast movement of the plasma (big arc current and argon flow rate), prevents the attachment from stopping at all accessible anode inhomogeneities and the dwelling frequency decreases (see Table 1). For the arc current around $400 \mathrm{~A}$, there is a local maximum in this frequency, what means more frequent dwelling of attachments and consequently stronger evaporation of the anode (characteristic dwell time remains nearly unchanged). It seems that the use of the hybrid plasma torches with some medium arc current value (in our case about 400 A) shortens the lifespan of the anode more than expected.

The dwellings of the attachments shorter than $4 \mu \mathrm{s}$ were difficult to examine because of the accuracy of the measured cathode-anode voltage approx. $0.4 \mathrm{~V}$ (the voltage signal was decreased $\times 1000)$ and the electrical noise in the range of around $\pm 0.3 \mathrm{~V}$. Thus, the movement of the attachment whilst increasing the voltage by the $0.4 \mathrm{~V}$ could not be investigated. For the attachment stop defined by a shorter minimum time (e.g. $2 \mu \mathrm{s}$ ), the characteristic dwell time would be shorter and characteristic dwelling frequency would be greater.

\section{Acknowledgements}

This work has been supported by the Grant Agency of the Czech Republic under the project GA15-19444S.

\section{References}

[1] Brezina V. New plasma spraying torch with combined gas-liquid stabilization of arc. In Proc. of the 15th Int. Symp. on Plasma Chem., Orleans, France, 3:1021-1026, 2001.

[2] Kavka T., Matejicek J., Ctibor P., Maslani A., and Hrabovsky M. Plasma spraying of copper by hybrid water-gas dc arc plasma torch. J. Therm. Spray Technol., 20:760-774, 2011.

[3] Hlina M., Hrabovsky M., Kavka T., and Konrad M. Production of high quality syngas from argon/water plasma gasification of biomass and waste. Waste management, 34:63-66, 2014.

[4] Kavka T., Chumak O., Sonsky J., Heinrich M., Stehrer T., and Pauser H. Experimental study of anode processes in plasma arc cutting. J. Phys. D: Appl. Phys., 46(0652502):11, 2013.

[5] Boselli M., Colombo V., Ghedini E., Gherardi M., and Sanibondi P. Two-dimensional time-dependent modelling of fume formation in a pulsed gas metal arc welding process. J. Phys. D: Appl. Phys., 46(224009):11, 2013.

[6] Eckert E. R. G., Pfender E., and Wutzke S. A. Study of electric arc behavior with superimposed flow. AIAA Journal, 5:707-713, 1967.

[7] Zhukov M. F. Applied dynamics of thermal plasma. Second printing. Izdatel'stvo Nauka, Novosibirsk, Russia, 1975.

[8] Hrabovsky M. Destruction of liquid organic compounds in hydrogen-oxygen thermal plasma jet. Progress in Plasma Processing of Materials, 1:1-8, 1997.

[9] Hrabovsky M., Konrad M., Kopecky V., Hlina J., Benes J., and Vesely E. Motion of anode attachment and fluctuations of plasma jet in dc arc plasma torch. High Temp. Mater. Processes, 1:167-168, 1997.

[10] Chumak O. M. Experimental observations of arc-anode attachment in steam-argon-air environment. Journal of Physics: Conference Series, 406(012009), 2012.

[11] Wutzke S. A. Conditions Governing the Symptomatic Behaviour of an Electric Arc in a Superimposed Flow Field. Ph.D. Thesis, University of Minnesota, 1967.

[12] Russ S. Anode arc attachment control using boundary layer bleed holes. Proc. of the 1993 NTSC, Anaheim, CA, pages 97-103, 1993.

[13] Kopecky V. and Hrabovsky M. Determination of flow velocity from analysis of hydrodynamic oscillations of thermal plasma jet. High Temp. Mater. Processes, $7: 23-28,2009$

[14] Kopecky V. Dependence of frequency and phase velocity of plasma jet hydrodynamic instability on sound velocity. Czech. J. Phys., 54:C1056-C1061, 2004.

[15] Hrabovsky M. Investigation of rayleigh instability in thermal plasma jet generated by plasma torch with external anode. Proc. of the15th Int. Symp. on Plasma Chem., Orleans, Gremi, CNRS University of Oreans, pages 861-866, 2001.

[16] Kopecky V. and Hrabovsky M. Resonant excitation of boundary layer instability of dc arc plasma jet by current modulation. Plasma Chem. Plasma Process., 31:827-838, 2011.

[17] Kavka T. Full-Papers CD 18th Int. Symp. on Plasma Chem., in Kyoto, Japan, ISBN 978-4-9903773-4-2, 2007. 